\title{
Three-Dimensional Pulse Width Modulation Techniques for Three-Phase Four Leg Voltage Source Converters
}

\author{
Mohamed ELBAR ${ }^{(1)}$, Imad MERZOUK ${ }^{(1)}$, Mohamed Mounir REZAOUI ${ }^{(1)^{*}}$, Noureddine BESSOUS ${ }^{(2)}$ \\ (1) LAADI, Faculty of Science and Technology, University of Djelfa 17000 DZ, Algeria \\ ${ }^{(2)}$ Faculty of Science and Technology, University of El Oued 39000 DZ, Algeria \\ *mm_rezaoui@mail.univ-djelfa.dz
}

\begin{abstract}
This paper presents a new control strategy to improve the performance of shunt active power filter (SAPF) in three-phase four-wire system when operating under an unbalanced system. A control method based on the instantaneous power theory have been used and discussed. On the other side, a threedimensional hysteresis current controller is used to track the compensated current references. This scheme requires the service of a phased-locked loop (PLL) to make the system insensitive to the distortions that are generally present in the utility voltages. The performance of the proposed topology under the used control approach has been finally discussed through the obtained simulation results.
\end{abstract}

Keywords: Active power filter, Three-phase four wire system, instantaneous power, $p q r$ control theory.

\section{INTRODUCTION}

A nonlinear load is an electrical device which draws current discontinuously or whose impedance varies throughout the cycle of the input ac voltage waveform. Nonlinear loads, which are mainly power electronics-based power converters, are known as the primary sources of harmonics in electric power systems. When a sinusoidal voltage with a constant frequency $(50 \mathrm{~Hz})$ is applied to a nonlinear load, such as switch-mode power supply, a distorted current waveform will be resulted. This distorted current contains the fundamental sinusoidal current plus a number of low order harmonic currents (3rd, 5th, 7th, etc.) at harmonic frequencies of $150 \mathrm{~Hz}$, $250 \mathrm{~Hz}, 350 \mathrm{~Hz}$, respectively [1].

Diode rectifiers and thyristor rectifiers are well-known types of nonlinear loads [2]. These devices are primarily used in power electronic applications, such as switch-mode power supplies in PCs, fax machines, and ASDs, and inject a large amount of harmonics into power systems. Three-phase nonlinear loads such as ASDs primarily inject nontriplen odd harmonics into power systems. Single-phase nonlinear loads such as switchmode power supplies inject all odd harmonics with the third harmonic being dominant. The triplen harmonics of single-phase nonlinear loads add up in the neutral conductor and cause excessive overheating problems in the neutral conductors and distribution transformers.

Active power filters for three-phase systems without neutral conductor have been successfully developed, and nowadays some commercial products are already available [3]. Although three-phase four-wire active filters have been introduced in the 1980's, the development is still in its infancy and no experimental prototype has been put in operation outside the universities [4] and in work of [5].

Some researches appoint the four-leg converter topology as the best alternative to implement a three-phase four-wire active power filter. In order to control the four-leg active filter based inverter, many control strategies were proposed. The four hysteresis comparator controls has been considered as a simplest control strategy but it suffers from a high frequency operation; generally the PWM is interested for the feeding and the control of induction electrical machine [6], particularly the three dimensional hysteresis PWM techniques presented in [7] uses eight switching vectors among the existing sixteen switching vectors.

This paper presents a study and analyses of a three dimensional hysteresis PWM techniques for three phases four legs used as an active shunt filter under unbalanced and non-sinusoidal conditions. An advanced control theory named $p q r$ theory has been already proposed by $\mathrm{H}$. Kim et al [8]. The $p q r$ theory takes the advantages of both $p q$ theory and cross vector theory. The defined instantaneous powers follow power conservation. Both instantaneous real and imaginary powers can be defined in the zero- 
sequence circuit in three-phase four-wire systems. The three power components are linearly independent of each other.

The proposed pqr theory shows superior aspects both in definition and compensation. When the system voltages are unbalanced or distorted by harmonics, the system currents can't be controlled balanced and sinusoidal even though the ac part of the powers and are compensated completely.

To overcome this problem, the $p q r$ theory need to preprocess the system voltages to be sinusoidal and balanced waveforms by using such as a PLL (phase locked loop) circuit [9], extracting only the forward-sequence component from the system voltages. Various circuit conditions are simulated to show the performance of the proposed compensation algorithm, such as unbalance/harmonic source voltages and nonlinear single-phase rectifier loads.

\section{INSTANTANEOUS pqr STRATEGY}

In this method, a set of voltages $\left(v_{a}, v_{b}, v_{c}\right)$ and currents $\left(i_{a}, i_{b}, i_{c}\right)$ from a three-phase four-wire system are first transformed into a three-axis representation, using the power invariant.

$$
\begin{gathered}
{\left[\begin{array}{l}
v_{o} \\
v_{\alpha} \\
v_{\beta}
\end{array}\right]=C\left[\begin{array}{l}
v_{a} \\
v_{b} \\
v_{c}
\end{array}\right] ;\left[\begin{array}{l}
i_{o} \\
i_{\alpha} \\
i_{\beta}
\end{array}\right]=C\left[\begin{array}{l}
i_{a} \\
i_{b} \\
i_{c}
\end{array}\right]} \\
\text { With: } C=\sqrt{\frac{2}{3}}\left[\begin{array}{ccc}
1 / \sqrt{2} & 1 / \sqrt{2} & 1 / \sqrt{2} \\
1 & -1 / 2 & -1 / 2 \\
0 & \sqrt{3} / 2 & -\sqrt{3} / 2
\end{array}\right]
\end{gathered}
$$

In Cartesian pqr coordinates, the system currents are defined in [8]. The $p q r$ coordinates are rotating along with the system voltage space vector.

$$
\left.\left[\begin{array}{c}
i_{p} \\
i_{q} \\
i_{r}
\end{array}\right]=\left[\begin{array}{ccc}
\frac{v_{\alpha}}{v_{\alpha \beta o}} & \frac{v_{\beta}}{v_{\alpha \beta o}} & \frac{v_{o}}{v_{\alpha \beta o}} \\
\frac{-v_{\beta}}{v_{\alpha \beta}} & \frac{v_{\alpha}}{v_{\alpha \beta}} & 0 \\
\frac{-v_{\alpha} v_{o}}{v_{\alpha \beta} v_{\alpha \beta o}} & \frac{-v_{\beta} v_{o}}{v_{\alpha \beta} v_{\alpha \beta o}} & \frac{v_{\alpha \beta}}{v_{\alpha \beta o}}
\end{array}\right] \mid \begin{array}{c}
i_{\alpha} \\
i_{\beta} \\
i_{o}
\end{array}\right]
$$

With:

$$
v_{\alpha \beta o}=\sqrt{v_{\alpha}^{2}+v_{\beta}^{2}+v_{o}^{2}}, v_{\alpha \beta}=\sqrt{v_{\alpha}^{2}+v_{\beta}^{2}}
$$

In $p q r$ coordinates, the system voltages are defined by (3).The voltage exists only in axis

$p$.

$$
\left[\begin{array}{c}
v_{p} \\
v_{q} \\
v_{r}
\end{array}\right]=\left[\begin{array}{ccc}
\frac{v_{\alpha}}{v_{\alpha \beta o}} & \frac{v_{\beta}}{v_{\alpha \beta o}} & \frac{v_{o}}{v_{\alpha \beta o}} \\
\frac{-v_{\beta}}{v_{\alpha \beta}} & \frac{v_{\alpha}}{v_{\alpha \beta}} & 0 \\
\frac{-v_{\alpha} v_{o}}{v_{\alpha \beta} v_{\alpha \beta o}} & \frac{-v_{\beta} v_{o}}{v_{\alpha \beta} v_{\alpha \beta o}} & \frac{v_{\alpha \beta}}{v_{\alpha \beta o}}
\end{array}\right]\left|\begin{array}{l}
v_{\alpha \beta o} \\
0 \\
0
\end{array}\right|
$$

The instantaneous active and reactive power will be defined by:

$$
\left[\begin{array}{l}
p \\
q_{q} \\
q_{r}
\end{array}\right]=\left|\begin{array}{l}
v_{p} i_{p} \\
-v_{p} i_{r} \\
v_{p} i_{q}
\end{array}\right|
$$

The three instantaneous powers are linearly independent of each other. Thus, the three current components can be controlled independently by compensating for the three instantaneous powers respectively. Moreover, each instantaneous power is defined in the same way as for single-phase systems.

In order to extract only the current harmonics, which will be injected by the APF, the continuous component, $p$, should be eliminated. Thus, the currents in $\alpha \beta$ o coordinates will become:

$$
\left|\begin{array}{c}
i_{f_{\alpha}}^{r e f} \\
i_{f \beta}^{r e f} \\
i_{f o}^{r e f}
\end{array}\right|=\frac{1}{v_{\alpha \beta o}}\left[\begin{array}{ccc}
\frac{v_{\alpha}}{v_{\alpha \beta o}} & \frac{v_{\beta}}{v_{\alpha \beta o}} & \frac{v_{o}}{v_{\alpha \beta o}} \\
\frac{-v_{\beta}}{v_{\alpha \beta}} & \frac{v_{\alpha}}{v_{\alpha \beta}} & 0 \\
\frac{-v_{\alpha} v_{o}}{v_{\alpha \beta} v_{\alpha \beta o}} & \frac{-v_{\beta} v_{o}}{v_{\alpha \beta} v_{\alpha \beta o}} & \frac{v_{\alpha \beta}}{v_{\alpha \beta o}}
\end{array}\right]\left|\begin{array}{l}
\tilde{p} \\
q_{r} \\
q_{q}
\end{array}\right|
$$

\section{IMPROVED THEORY}

The main drawback of this theory is when the voltages in the electrical network are disturbed by harmonics and/or unbalanced. To overcome this problem, this paper proposes to use a PLL in the control loop [9]. The PLL generates balanced and sinusoidal signals, perfectly in phase with the electrical network voltages. They will be directly generated in the $\alpha \beta$ co-ordinates illustrated by the following expressions:

$$
\begin{aligned}
& v_{\alpha}=\sqrt{3} V_{s} \sin (\omega t) \\
& v_{\beta}=-\sqrt{3} V_{s} \cos (\omega t)
\end{aligned}
$$


Equations (2) and (6) give:

$$
\left[\begin{array}{l}
i_{p} \\
i_{q} \\
i_{r}
\end{array}\right]=\left[\begin{array}{ccc}
\sin (\omega t) & -\cos (\omega t) & 0 \\
\cos (\omega t) & \sin (\omega t) & 0 \\
0 & 0 & 1
\end{array}\right]\left|\begin{array}{l}
i_{\alpha} \\
i_{\beta} \\
i_{o}
\end{array}\right|
$$

Then the instantaneous active and reactive powers will be:

$$
\left[\begin{array}{c}
p \\
q_{q} \\
q_{r}
\end{array}\right]=\sqrt{3} V_{s}\left[\begin{array}{lll}
1 & 0 & 0 \\
0 & 1 & 0 \\
0 & 0 & 1
\end{array}\right]\left|\begin{array}{c}
i_{p} \\
i_{q} \\
i_{r}
\end{array}\right|
$$

In $\alpha \beta O$ co-ordinates the currents become:

$$
\left|\begin{array}{l}
i_{f_{\alpha}^{r e f}}^{r} \\
i_{f \beta}^{r e f} \\
i_{f o f}^{r e f}
\end{array}\right|=\left[\begin{array}{ccc}
\sin (\omega t) & \cos (\omega t) & 0 \\
-\cos (\omega t) & \sin (\omega t) & 0 \\
0 & 0 & 1
\end{array}\right]\left|\begin{array}{l}
\tilde{p} \\
q_{r} \\
q_{q}
\end{array}\right|
$$

\section{DIMENSIONAL HYSTERESIS PWM TECHNIQUE}

The power quality is insured by using threelevel inverter with several modulation techniques in series with filter [10].

In a three-legged inverter, there are eight possible switch combinations. With the fourth neutral leg, the total number of switch combinations increases to sixteen. The switch combinations are represented by ordered sets $\left[S_{a}, S_{b}, S_{c}, S_{\mathrm{n}}\right]$, where $S_{a}=' p$ ' denotes that the upper switch in phase $\mathrm{A}, S_{a p}$, is closed, and $S_{a}=' n$ ' denotes that the bottom switch in phase A, $S_{a n}$, is closed. The same notation applies to phase legs $B$ and $C$ and the fourth neutral leg. The terminal voltages $\left[v_{a n}, v_{b n}, v_{c n}\right]^{T}$ in $a b c$ coordinate can be transformed into $\left[v_{\alpha}, v_{\beta}, v_{o}\right]^{T}$ in $\alpha \beta o$ orthogonal coordinate. The results of the transformation are shown in Table1. There are fourteen non-zero space voltage vectors (NZSVV) and two zero space voltage vectors (ZSVV) as shown in Fig1 [11-13]

The reference current obtained by (9) can be written with the space vector representation as follows:

$$
\vec{i}_{f}^{r e f}=i_{f_{\alpha}}^{r e f} \vec{i}+i_{f \beta}^{r e f} \vec{j}+i_{f o}^{r e f} \vec{k}
$$

The same representation can be given to the measured active filter current:

$$
\vec{i}_{f}=i_{f_{\alpha}} \vec{i}+i_{f \beta} \vec{j}+i_{f o} \vec{k}
$$

The reference current is represented in space voltage vector as shown in Fig 2, the tip of the reference current $\vec{i}_{f}^{\text {ref }}$ is located at the centre of a three dimensional cube, while the tip of the measured active filter current $\vec{i}_{f}$ can be located in any cube of the eight small cubes as illustrated in Fig 3.



Fig. 1 Switching vectors in $\alpha \beta o$ coordinate.

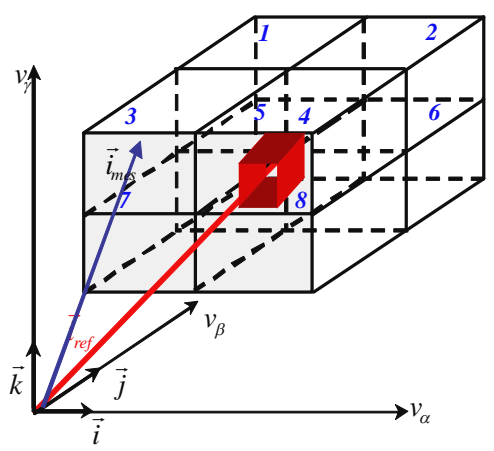

Fig. 2 Reference current vector representation in $\alpha \beta o$ plane

The error between the reference current and the active filter current, at any sampling time $t$ can be expressed as follows:

$$
\left[\begin{array}{c}
\varepsilon_{\alpha} \\
\varepsilon_{\beta} \\
\varepsilon_{o}
\end{array}\right]=\left[\begin{array}{c}
i_{f \alpha}^{r e f}(k)-i_{f \alpha}(k) \\
i_{f \beta}^{r e f}(k)-i_{f \beta}(k) \\
i_{f o}^{r e f}(k)-i_{f o}(k)
\end{array}\right]
$$

The eight cubes can be determined according to the sign of the calculated error as shown in Fig3. 
In order to minimise the error, a suitable space voltage vector among the sixteen space voltage vectors should be applied to force the measured vector to be as close as possible to coincide with the reference vector within the hysteresis boundary.

Table 1. Switch Combinations in $\alpha \beta$ o orthogonal coordinate

\begin{tabular}{|l|c|c|c|c|c|c|c|c|}
\cline { 2 - 8 } \multicolumn{1}{l|}{} & $\mathrm{pppp}$ & $\mathrm{nnnp}$ & $\mathrm{pnnp}$ & $\mathrm{ppnp}$ & $\mathrm{npnp}$ & $\mathrm{nppp}$ & $\mathrm{nnpp}$ & $\mathrm{pnpp}$ \\
\hline$v_{\alpha}$ & 0 & 0 & $\frac{2 v_{c}}{3}$ & $\frac{v_{c}}{3}$ & $\frac{-v_{c}}{3}$ & $\frac{-2 v_{c}}{3}$ & $\frac{-v_{c}}{3}$ & $\frac{v_{c}}{3}$ \\
\hline$v_{\beta}$ & 0 & 0 & 0 & $\frac{v_{c}}{\sqrt{3}}$ & $\frac{v_{c}}{\sqrt{3}}$ & 0 & $\frac{-v_{c}}{\sqrt{3}}$ & $\frac{-v_{c}}{\sqrt{3}}$ \\
\hline$v_{o}$ & 0 & $-v_{c}$ & $\frac{-2 v_{c}}{3}$ & $\frac{-v_{c}}{3}$ & $\frac{-2 v_{c}}{3}$ & $\frac{-v_{c}}{3}$ & $\frac{-2 v_{c}}{3}$ & $\frac{-v_{c}}{3}$ \\
\hline$v_{\alpha}$ & 0 & 0 & $\frac{2 v_{c}}{3}$ & $\frac{v_{c}}{3}$ & $\frac{-v_{c}}{3}$ & $\frac{-2 v_{c}}{3}$ & $\frac{-v_{c}}{3}$ & $\frac{v_{c}}{3}$ \\
\hline$v_{\beta}$ & 0 & 0 & 0 & $\frac{v_{c}}{\sqrt{3}}$ & $\frac{v_{c}}{\sqrt{3}}$ & 0 & $\frac{-v_{c}}{\sqrt{3}}$ & $\frac{-v_{c}}{\sqrt{3}}$ \\
\hline$v_{o}$ & $v_{c}$ & 0 & $\frac{v_{c}}{3}$ & $\frac{2 v_{c}}{3}$ & $\frac{v_{c}}{3}$ & $\frac{2 v_{c}}{3}$ & $\frac{v_{c}}{3}$ & $\frac{2 v_{c}}{3}$ \\
\hline
\end{tabular}



Cube 2 Cube 6 Cube 4 Cube 8<smiles>O=[N+]1CC2CCCC1C2</smiles>

Fig. 3 Detection of the cube

\begin{tabular}{|c|c|c|c|c|c|c|c|c|c|c|}
\hline & & & Cube 1 & Cube2 & Cube3 & Cube4 & Cube5 & Cube6 & Cube7 & Cube8 \\
\hline \multirow{4}{*}{$\varepsilon_{o}<h$} & \multirow[b]{2}{*}{$\varepsilon_{\beta}<h$} & $\varepsilon_{\alpha}<h$ & npnn & ppnn & nnpn & pnpn & npnp & ppnp & nnpp & pnpp \\
\hline & & $\varepsilon_{\alpha}>h$ & npnn & ppnn & nnpn & pnpn & npnp & ppnp & nnpp & pnpp \\
\hline & \multirow[b]{2}{*}{$\varepsilon_{\beta}>h$} & $\varepsilon_{\alpha}<h$ & npnn & ppnn & nnpn & pnpn & npnp & ppnp & nnpp & pnpp \\
\hline & & $\varepsilon_{\alpha}>h$ & npnn & ppnn & nnpn & pnpn & npnp & ppnp & nnpp & pnpp \\
\hline \multirow{4}{*}{$\varepsilon_{o}>h$} & \multirow[b]{2}{*}{$\varepsilon_{\beta}<h$} & $\varepsilon_{\alpha}<h$ & npnn & ppnn & nnpn & pnpn & npnp & ppnp & nnpp & pnpp \\
\hline & & $\varepsilon_{\alpha}>h$ & npnn & ppnn & nnpn & pnpn & npnp & ppnp & nnpp & pnpp \\
\hline & \multirow[b]{2}{*}{$\varepsilon_{\beta}>h$} & $\varepsilon_{\alpha}<h$ & npnn & ppnn & nnpn & pnpn & npnp & ppnp & nnpp & pnpp \\
\hline & & $\varepsilon_{\alpha}>h$ & npnn & ppnn & nnpn & pnpn & npnp & ppnp & nnpp & pnpp \\
\hline
\end{tabular}


$\left(\varepsilon_{\alpha}(k) \cdot \frac{d \varepsilon_{\alpha}(k)}{d t}<0, \varepsilon_{\beta}(k) \cdot \frac{d \varepsilon_{\beta}(k)}{d t}<0, \varepsilon_{o}(k) \cdot \frac{d \varepsilon_{o}(k)}{d t}<0\right)(13$

Moreover the two space voltage vectors $\vec{v}$ (nnnp) and $\vec{v}$ (pppn), which can be considered as a ZSVV regarding to their influence on $\varepsilon_{\alpha}(k)$ and $\varepsilon_{\beta}(k)$, can be applied when it is certain that only the errors $\varepsilon_{\alpha}(k)$ and $\varepsilon_{\beta}(k)$ have the tendency to converge toward the hysteresis boundary and the $\varepsilon_{o}(k)$ has the tendency to diverge from the hysteresis boundary. Similarly, this case can be verified by satisfying the following condition:

$$
\left(\varepsilon_{\alpha}(k) \cdot \frac{d \varepsilon_{\alpha}(k)}{d t}<0, \varepsilon_{\beta}(k) \cdot \frac{d \varepsilon_{\beta}(k)}{d t}<0, \varepsilon_{o}(k) \cdot \frac{d \varepsilon_{o}(k)}{d t}>0\right)
$$

However; when the equations (13) and (14) are both not verified, in other words when the current error components and their respective derivative in $\alpha \beta o$ reference frame have the same sign; it is necessary to apply the suitable non-zero space voltage vector to force the measured active filter current to forward the right trajectory towards the hysteresis boundary.

For each of the eight possible locations, shown in Fig2, there are eight space voltage vectors which can be applied, among them: one of either ZSVV, which can be chosen as $\vec{v}$ (nnnn) or $\vec{v}$ (pppp) in the case when condition (13) is verified. Similarly one ZSVV which can be applied as $\vec{v}$ (nnnp) or $\vec{v}$ (pppn), in the case when equation (14) is verified, and six active NZSVV.

In the same cube, the eight space voltage vectors can drive the measured active filter current vector towards the reference current vector with reducing the switching frequency. Sixty four (64) possible configurations can be distinguished, summarising the eight cubes. Table3 shows the complete look up table implemented to control the filter current in three phase four wires active filter.

\section{SIMULATIONS AND RESULTS}

In order to predict the performance of the APF using a four leg converter, detailed simulation studies on Matlab/Simulink platform are carried out. A $220 \mathrm{~V}, 50 \mathrm{~Hz}$ three phase four wire system is chosen for this study. A three single phase diode rectifiers feeding unbalanced load are connected in phases and to the neutral, respectively. The reference dc link voltage of the converter is maintained at $700 \mathrm{~V}$. The current THD calculated for the 30 first harmonics before filtering is $40.7 \%$. The converter ac currents are controlled with the proposed control theory. The current controller switching table 2 is considered. In order to show the good dynamic performance of the current controller, some simulation results are presented in the next figures.

Fig.4 shows waveforms of the system before and after instantaneous reactive power compensation, where the source voltage is balanced and has no zero-sequence components and the load current contains zero-sequence component. It could be remarked that the filter dc voltage converges to its desired value and the line currents are practically sine waves and in phase with their corresponding voltages at the PCC. The current THD has dropped to $1.5 \%$ (less than $5 \%$ IEC norm), and the source power factor at the PCC has increased to 1.

The second simulations, Fig.5, give the currents before and after instantaneous reactive power compensation, when the lines voltages are unbalanced, up to $5 \%$ but do not contain harmonics. It could be remarked that the filter dc voltage converges to its desired value and the line currents are practically sine waves and in phase with their corresponding voltages at the PCC. The current THD has dropped to $1.8 \%$ (less than 5\% IEC norm), and the source power factor at the PCC has increased to 1



Fig. 4 Simulation Waveforms under balanced supply network. 


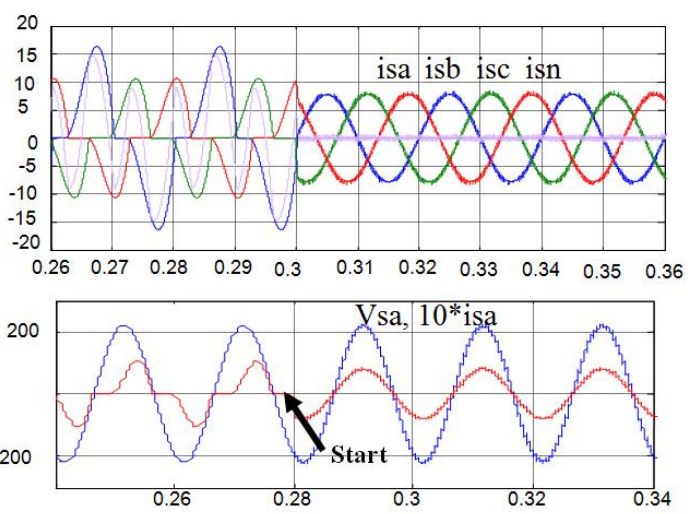

Fig. 5 Simulation Waveforms under unbalanced supply network.

\section{CONCLUSION}

In this paper, three dimensional hysteresis PWM techniques is proposed, it has main advantage over the hysteresis control method where it groups the four errors into one vector error. It has also another advantage over the double hysteresis control where it has less switches losses due to the application of zero voltage space vectors. pqr theory is mathematically clear and systematic, showing good compensating characteristics. The equivalent circuit valid for pqr theory is very simple and it gives clear physical meaning.

The active power filter based on the proposed control strategy can compensate unbalanced non-linear load currents even the source voltages are unbalanced and distorted. The effectiveness of the proposed scheme was evaluated through digital simulations. The active filter has good performance under unbalanced load conditions

\section{References}

[1] Liu C, Dai K, Chen X,; He Y, Lu P. Selective harmonic suppression strategy by SAPF in power distribution system. The Journal of Engineering. 2017; 17(13): 1610-1613.

[2] Peter ES, Barry B. History of Harmonics History. IEEE Industry Applications Magazine. 2015; 21(3): 6-11.

[3] Lopez J, Fabian R, Pinzon T, Andres C, Edwin F. Current and voltage harmonics acquisition and obtaining reference currents for an Single Phase shunt Active Power Filter. IEEE Workshop on Power Electronics and Power Quality Applications (PEPQA) - Bogota DC, Colombia. 2013, pp.1-8.

[4] Tan KCA, Cuma BMU, Tumay M. Multiple harmonic élimination-based feedback controller for Shunt Hybrid Active Power Filter. IET Power Electronics. 2017, pp. 945956.
[5] Adel Dahdouh, Said Barkat, Aissa Chouder, "A Combined Sliding Mode Space vector Modulation Control of the Shunt Active Power Filter Using Robust Harmonic Extraction Method ", Algerian Journal of Signals and Systems, Vol.1, Issue1, June 2016. pp:37-46.

[6] Ghania Aggoun, Naima Boubouzel, Rahma Kachenoura, Sid Ali Fellag, "Quality of energy resulting from association converter- machine for the electric drive of a system of wind generation"', Algerian Journal of Signals and Systems, Vol.2, Issue1, April 2017. pp:1-11

[7] Ruderman A. About Voltage Total Harmonic Distortion for Single and Three-Phase Multilevel Inverters. IEEE Transactions on Industrial Electronics. 2015; 62(3):1548-1551.

[8] Amamra SA, Meghriche K, Cherifi A, Bruno F. Multilevel Inverter Topology for Renewable Energy Grid Integration. IEEE Transactions on Industrial Electronics. 2017; 64(11): 8855 8866.

[9] Ayhan O, Zekeriya O, Digital current control of a three phase four-leg voltage source inverter by using p-q-r theory. IET Power Electronics. 2014; 7(3): 527-539.

[10] Yacine AYACHI AMOR, Farid HAMOUDI Aissa KHELDOUN, "Three-phase Three-level Inverter Grid-tied PV System with Fuzzy Logic Control based MPPT", Algerian Journal of Signals and Systems, Vol.3, Issue 3, September 2018. pp:96-105

[11] Aredes M, Akagi H, Watanabe ES, Vergara E, Encarnacao L. Comparisons Between the $p-q$ and $p-q-r$ Theories in Three-Phase FourWire Systems. IEEE Transactions on Power Electronics. 2009; 24(4):924-933.

[12] Chung-Chuan $H$, Yung-Fu $H$. Design of single-phase shunt active filter for threephase four-wire distribution systems. IEEE Energy Conversion Congress and Exposition (ECCE) - Atlanta, GA, USA. 2010, pp. 15251528.

[13] Guangyu S, Yongli L, Wei J, Lizhi B. A Nonlinear Three-Phase Phase-Locked Loop Based on Linear Active Disturbance Rejection Controller. IEEE Access. 2017; 5: 2154821556. 